\title{
Iceberg Detection in Open Water and Sea Ice using C-Band Radar Polarimetry
}

\author{
Vahid Akbari and Camilla Brekke \\ Earth Observation Laboratory \\ Department of Physics and Technology \\ UiT-The Arctic University of Norway
}

\begin{abstract}
Icebergs can cause a significant danger for shipping, offshore oil exploration, and undersea or subsea pipelines and production facilities. Synthetic aperture radar (SAR) is very valuable tool of detecting and monitoring icebergs in the often dark and cloud-covered polar regions. Detection of small icebergs floating in nonhomegeous sea clutter environments is a challenging task in remote sensing. In this paper, a new methodology is proposed for automatic identification of icebergs in highresolution polarimetric SAR images acquired during different seasons. This involves adapting the algorithm to sea-ice conditions, and facing challenges when it comes to high iceberg density, meteorological and oceanographic phenomena in the marginal ice zone causing heterogeneity in the background clutter. The algorithm is tested with time series of RADARSAT-2 C-band quad-polarimetric images to detect icebergs in Kongsfjorden ( $\mathrm{Ny}$ Ålesund, Svalbard) that have broken off from the glaciers nearby.

Index Terms-Iceberg, sea ice, synthetic aperture radar, polarimetry.
\end{abstract}

\section{INTRODUCTION}

As icebergs represent a significant hazard for shipping and offshore oil and gas exploration or production, any improvement in the ability to detect and track their progress along the Arctic regions would be valuable. Since spaceborne synthetic aperture radar (SAR) systems can image the ocean and their sea ice regions independent of light and cloud conditions, its potential for iceberg monitoring is a relevant application of remote sensing.

The potential of SAR remote sensing for iceberg detection depends on several factors: 1) physical properties of the iceberg itself, such as size, shape, and structure; 2) SAR sensor specific properties, such as incidence and azimuth angles, resolution, frequency, and polarization; 3) geophysical parameters, such as winds, sea state, surface currents, temperature, seasons, and 4) the backscatter of the surrounding sea ice or open water. Many studies have demonstrated the potential of SAR images in icebergs detection and characterization, e.g., [1]-[3]. This study is devoted to the polarimetric SAR (PolSAR) sensor, which potentially provides increased detection capability, as compared to single-polarization SAR [4].

The well-known constant false alarm rate (CFAR) target detectors adaptively determine the detection threshold based on accurate statistical modeling of the local background clutter measurements [5]. The CFARs are often implemented with the sliding window technique, and the parameters of the hypothesized model are estimated within this local reference window. In practice, however, even with a homogeneous background, the sea clutter confined by the standard sliding window is often contaminated, i.e., where nearby targets can reduce the sensitivity by contaminating the estimation window [6]. One common source of contamination is in areas of high iceberg density which usually occurs at the tidewater glacier fronts. When there are too many icebergs in the local reference window, no longer statistical modeling of the sea clutter is estimated, but of a mixture of sea clutter and icebergs [7], which can result in severe degradation of the CFAR detector performance causing missed icebergs and an increased number of false alarms. In target detection context, this is generally knows as the capture effect [6], [8].

Another challenge with iceberg detection is heterogeneous clutter that is caused by background intensity transition due to meteorological and oceanic phenomena, e.g., transitions between regions with different wind conditions, low-wind spiral marks, and surrounding sea ice. They are reffered to be the clutter edge effect in [9], [10]. This challenge rises the number of false alarms and lowers the reliability of a automatic detection result. In addition, the CFAR technique is adopted to identify bright targets against a sea-clutter background, as long as these targets are smaller than the sliding window used. On the other hand, the larger icebergs vary widely in size and are frequently embedded in sea ice background, specially in winter.

In summary, in situations, with high iceberg density, heterogeneous background clutter, and icebergs varying with different sizes or embedded in sea ice, the standard CFAR detector reaches its limits. To tackle all these challenging tasks, a segmentation-based detection algorithm is proposed based on polarimetric SAR data. The algorithm uses a simple and fast feature-based segmentation algorithm to produce distinct, homogenous regions corresponding to ice and water from which different attributes such as area and shape can be determined. The proposed detector makes use of different polarized channels. The use of different polarizations is expected to add information because different targets exhibit different polarimetric behaviors. Therefore, the differences between clutter and targets can be magnified based on the responses at different polarizations, which helps detection or classification. The segmentation returns many clusters with high and lowintensities, but not all are icebergs. An identification step uses the clustering results to select the ones corresponding 
to icebergs. Compared to the conventional CFAR detector, the proposed iceberg detector has some advantages as: 1) it owns strong decision information because it models both clutter and the targets, whilst CFAR only models the background clutter; 2) It can handle different sea stages and sea-ice target situations automatically through segmentation, without needing window processing and tuning parameters of CFAR, i.e., target, buffer, and background windows; 3) pixelwise segmentation automatically handles high iceberg density situations.

The usefulness and effectiveness of the proposed method for detection of small icebergs are explored using full polarimetric data from RADARSAT-2 (RS-2) in Kongsfjorden (NyÅlesund, Svalbard) under different wind conditions and open water and/or sea-ice target situations.

\section{Methodology for Iceberg Detection}

The workflow of the proposed methodology for iceberg detection is shown in Fig. 1. The iceberg detection operation consists of the five major operations involved, i.e., the preprocessing, the land-sea masking, the polarimetric feature extraction, the segmentation, and the identification, which are sequentially explained in detail hereinafter. The simple nature of the proposed method means that a new scene can be imported, preprocessed, features extracted, segmented, and icebergs identified in less than half an hour and therefore falls well within the near real-time processing desired for operational use.

\section{A. Prepocessing}

The process begins with an input multi-channel single look complex (SLC) PolSAR data. The first step of the proposed methodology involves standard preprocessing (including calibration and geo-referencing). The raw SLC data are geocoded and multilooked simultaneously to a final geocoded resolution.

\section{B. Land-sea masking}

A binary mask using high resolution land boundary map is produced to mask out land areas from further analysis to ensure that detection is focused only on the area of interest and to avoid false alarms caused by strong radar returns from the land.

\section{Polarimetric Feature Selection}

A number of intensity and multipolarization SAR features are extracted from the the sample covariance matrix. These features are directly related to the physical properties of the scattering medium. These features are chosen based on the observation that icebergs or thick/deformed sea ice exhibits a different polarimetric behaviour compared to thinner sea ice or open water.

\section{Segmentation}

The objective of image segmentation in this step is to segment the image into distinct regions in non-homogenous sea clutter environments. It is important to note here that the segmentation process is one of the essential parts of

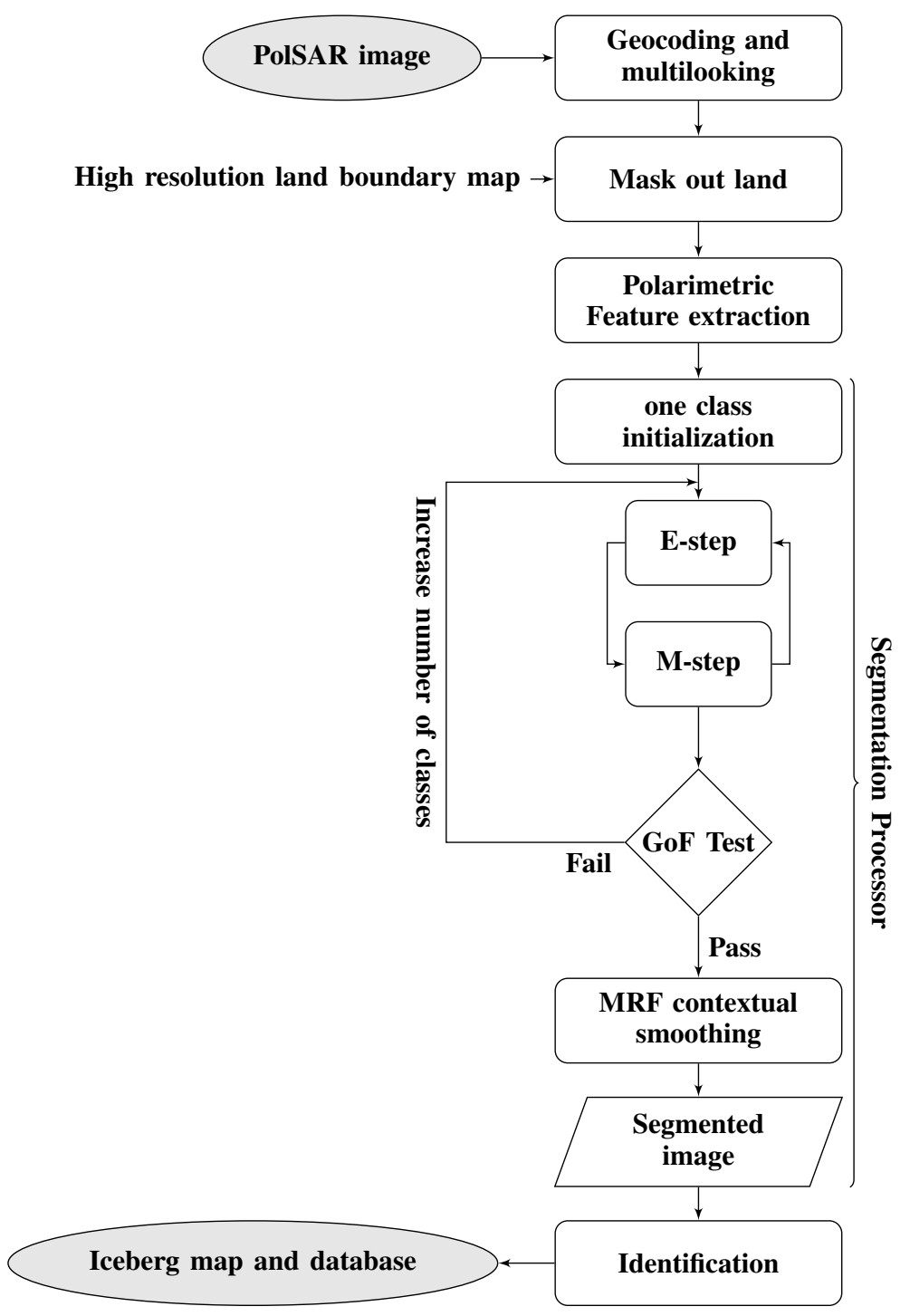

Fig. 1. Methodology for near real-time iceberg detection processing chain based on polarimetric SAR images.

the methodology and affects the overall performance of the methodology, therefore here we used an already established methodology reported in [11]. We aim to use a simple featurebased PolSAR segmentation that produces good, smooth, and robust results.

The advantage of this approach is its simplicity and speed, being able to segment full scenes into meaningful class segments in a matter of minutes, and is crucial for any algorithm supporting operational, near real time services. The automatic segmentation is achieved through a finite mixture model estimated with a Expectation Maximisation (EM) algorithm [12], where the number of clusters is automatically determined with the goodness-of-fit (GoF) based approach. The initial state and the number of classes are addressed with an automatic strategy by consistently starting as one class and adaptively splitting classes until a statistical criterion is satisfied in the GoF test. In addition, Markov random field (MRF) based contextual 
smoothing [13], [14] is applied after clustering to improve the quality and smoothness of the final image.

\section{E. Identification}

The segmentation of a PolSAR image results in a vast number of segments, both dim and bright, corresponding to homogeneous regions in the open water, sea ice, or icebergs/growlers. The identification step uses the segmentation result to select the segments corresponding to icebergs. A simple rule classifier is used to identify icebergs by applying a set of criteria that define typical ranges of geometrical parameters based on area, perimeter and major/minor axis.

Each segment is classified as an iceberg if all of the discrimination criteria fall within the ranges specified by user. The output of the identification step that undergoes iceberg detection is a binary image where each pixel is considered either a part of an iceberg or a part of the background clutter.

Subsequently the iceberg pixels are polygonized and various shape properties such as area, perimeter, major and minor axis length of each iceberg is calculated. Eventually, properties of icebergs and growlers are made available in tabular form.

\section{EXPERIMENTAL RESUlts}

In order to increase the probability of observing icebergs and growlers, the data were acquired over Kongsfjorden in $\mathrm{Ny}$-Ålesund, Svalbard. It is noted that two tidewater glaciers Kronebreen/Kongsbreen terminating in Kongsfjorden are capable of delivering small icebergs or bits to the fjord. The criterion for selection was to cover different environmental conditions such as freezing and melting, and different sea-ice types. Changing conditions affect the absolute radar intensities as well as the relative intensity contrast between the icebergs and the surrounding sea-ice or water surface. The inner part of Kongsfjorden is usually influenced by sea ice most of the year. The outer part of the fjord was either ice-free or covered with drift ice. Consequently these are the months where icebergs may drift around in the open sea. In addition, icebergs and ice pieces from surrounding glaciers can be found frozen into the fast ice or drifting in open water. The latter is a typical situation once all fast ice disappears in summer.

The satellite data consists of 14 RS-2 Fine Quad-pol scenes from both ascending and descending orbits over a range of incidence angles from $23^{\circ}$ to $45^{\circ}$. The images were delivered in a SLC slant-range format which are characterized by a nominal pixel spacing of $5.2 \mathrm{~m} \times 7.6 \mathrm{~m}$ in slant range and azimuth, respectively, covering approximately $25 \mathrm{~km} \times 25$ $\mathrm{km}$. RS-2 data containing icebergs and growlers in open water and/or sea ice background with different sea states are used to evaluate the performance of the iceberg detection algorithm described in the Section II. As examples of RS-2 scenes are illustrated in Fig. 2. The inner part of the fjord contain typical features of nonhomogeneities in the background clutter, i.e., high iceberg density, the clutter edge effect, mixture of open water and sea ice in the background clutter.

The SAR images were georeferenced and calibrated to produce MLC images with $20 \mathrm{~m}$ resolution and 16 looks. Since we focus on ocean regions, the calibration of the SAR images did not include terrain correction. Because icebergs in the open water and/or sea ice are of interest, land areas must be masked out of the SAR image to avoid false alarms. Land masking has been performed with the help of auxiliary data produced by the Mapping Section of the Norwegian Polar Institute (NPI). Fig. 3 shows the Pauli images, segmentation results, detection results, and icebergs centroids within the subsections (green dashed rectangle) in Fig. 2

\section{CONCLUSION}

In this paper, we proposed a processing chain for iceberg detection using high-resolution C-band polarimetric SAR data. We have looked into iceberg detection in nonhomogeneous sea clutter environments. A segmentation-based iceberg detection methodology has been proposed, where the introduction of the polarimetric features and the segmentation stage have proved to be beneficial in various situations. We tested the algorithm with a series of quad-polarimetric RS-2 images covering different sea states, wind conditions, and incidence angles in open water and sea ice background and high iceberg density areas.

\section{REFERENCES}

[1] D. Power, J. Youden, K. Lane, C. Randell, and D. Flett, "Iceberg detection capabilities of radarsat synthetic aperture radar," Canadian Journal of Remote Sensing, vol. 27, no. 5, pp. 476-486, 2001.

[2] C. Wesche and W. Dierking, "Iceberg signatures and detection in SAR images in two test regions of the Weddell sea, Antarctica," J. Glaciol., vol. 58, no. 208, pp. 325-339, 2012.

[3] R. Fenger-Nielsen, "Iceberg detection using synthetic aperture radar," Ph.D. dissertation, University of Copenhagen, April 2014.

[4] W. Dierking and C. Wesche, "C-band radar polarimetry: Useful for detection of icebergs in sea ice?" IEEE Trans. Geosci. Remote Sens., vol. 52, no. 1, pp. 25-37, Jan 2014.

[5] C. Oliver and S. Quegan, Understanding Synthetic Aperture Radar Images, 2nd ed. Raleigh, USA: SciTech Publishing, 2004.

[6] D. Tao, S. N. Anfinsen, and C. Brekke, "Robust CFAR detector based on truncated statistics in multiple-target situations," IEEE Trans. Geosci. Remote Sens., vol. 54, no. 1, pp. 117-134, Jan 2016.

[7] A. Frost, R. Ressel, and S. Lehner, "Automated iceberg detection using high-resolution X-band SAR images," Canadian Journal of Remote Sensing, vol. 42, no. 4, pp. 354-366, 2016.

[8] E. K. Al-Hussaini and B. M. Ibrahim, "Comparison of adaptive cellaveraging detectors for multiple-target situations," Communications, Radar and Signal Processing, IEE Proceedings F, vol. 133, no. 3, pp. 217-223, June 1986.

[9] P. Lombardo and M. Sciotti, "Segmentation-based technique for ship detection in sar images," IEE Proceedings - Radar, Sonar and Navigation, vol. 148, no. 3, pp. 147-159, Jun 2001.

[10] D. Tao, A. P. Doulgeris, and C. Brekke, "A segmentation-based CFAR detection algorithm using truncated statistics," IEEE Trans. Geosci. Remote Sens., vol. 54, no. 5, pp. 2887-2898, May 2016.

[11] A. P. Doulgeris, "A simple and extendable segmentation method for multi-polarisation SAR images," in in POLinSAR 2013, 2013, p. 8 pp.

[12] A. P. Dempster, N. M. Laird, and D. B. Rubin, "Maximum likelihood from incomplete data via the EM algorithm," Journal of the Royal Statistical Society, Series B, vol. 39, no. 1, pp. 1-38, 1977.

[13] L. S. Li, Markov Random Field Modeling in Image Analysis, 3rd ed. London, U.K.: Springer-Verlag, 2006.

[14] V. Akbari, A. P. Doulgeris, G. Moser, T. Eltoft, S. N. Anfinsen, and S. B. Serpico, "A textural -contextual model for unsupervised segmentation of multipolarization synthetic aperture radar images," IEEE Transactions on Geoscience and Remote Sensing, vol. 51, no. 4, pp. 2442-2453, April 2013. 

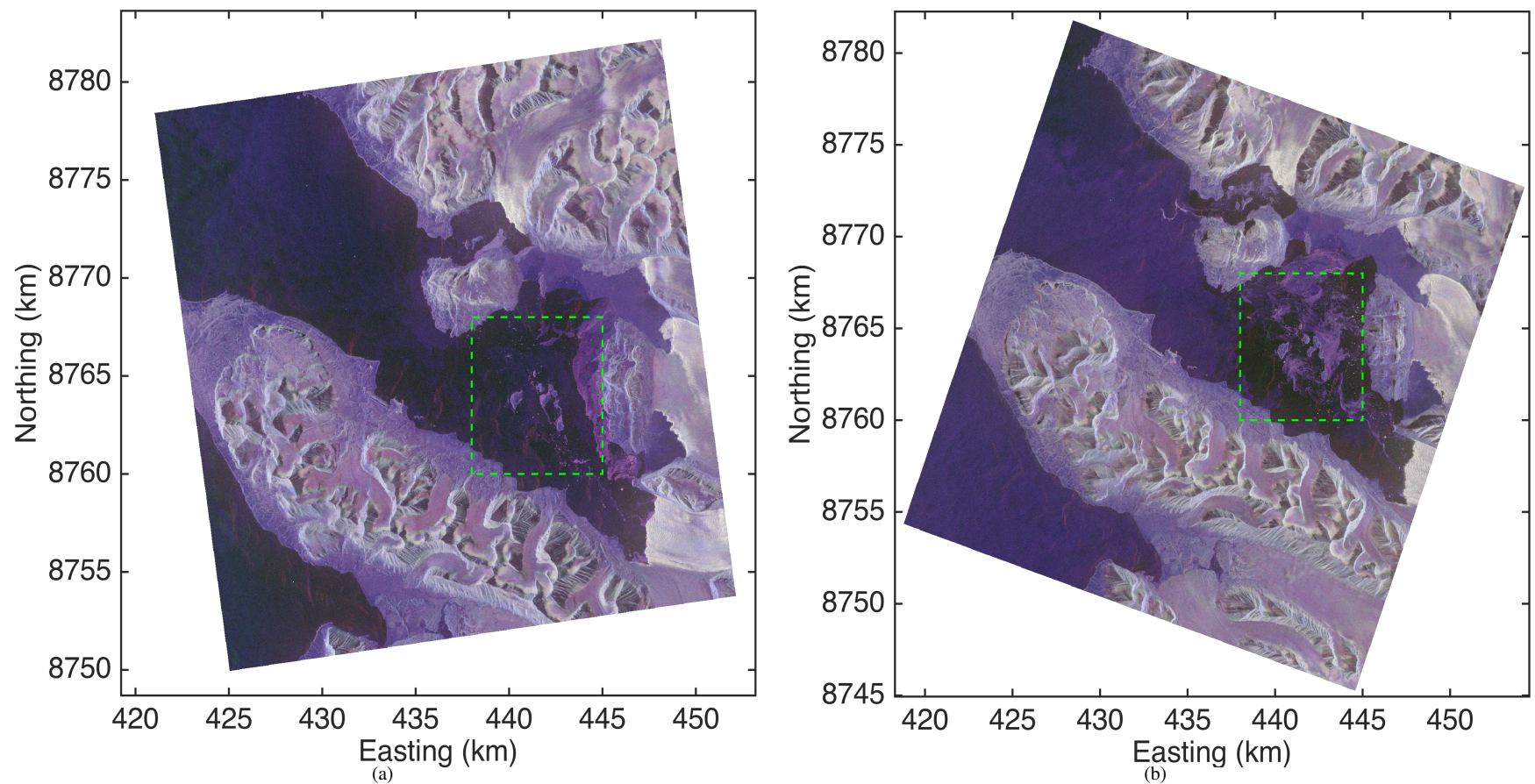

Fig. 2. Geocoded Pauli RGB composite ( $\mathrm{R}=\mathrm{HH}-\mathrm{VV}, \mathrm{G}=\mathrm{HV}, \mathrm{B}=\mathrm{HH}+\mathrm{VV}$ ) of the quad-polarimetric RS-2 acquisitions on April 19, 2015 (left), April 20, 2015 (right) multilooked with 16-looks
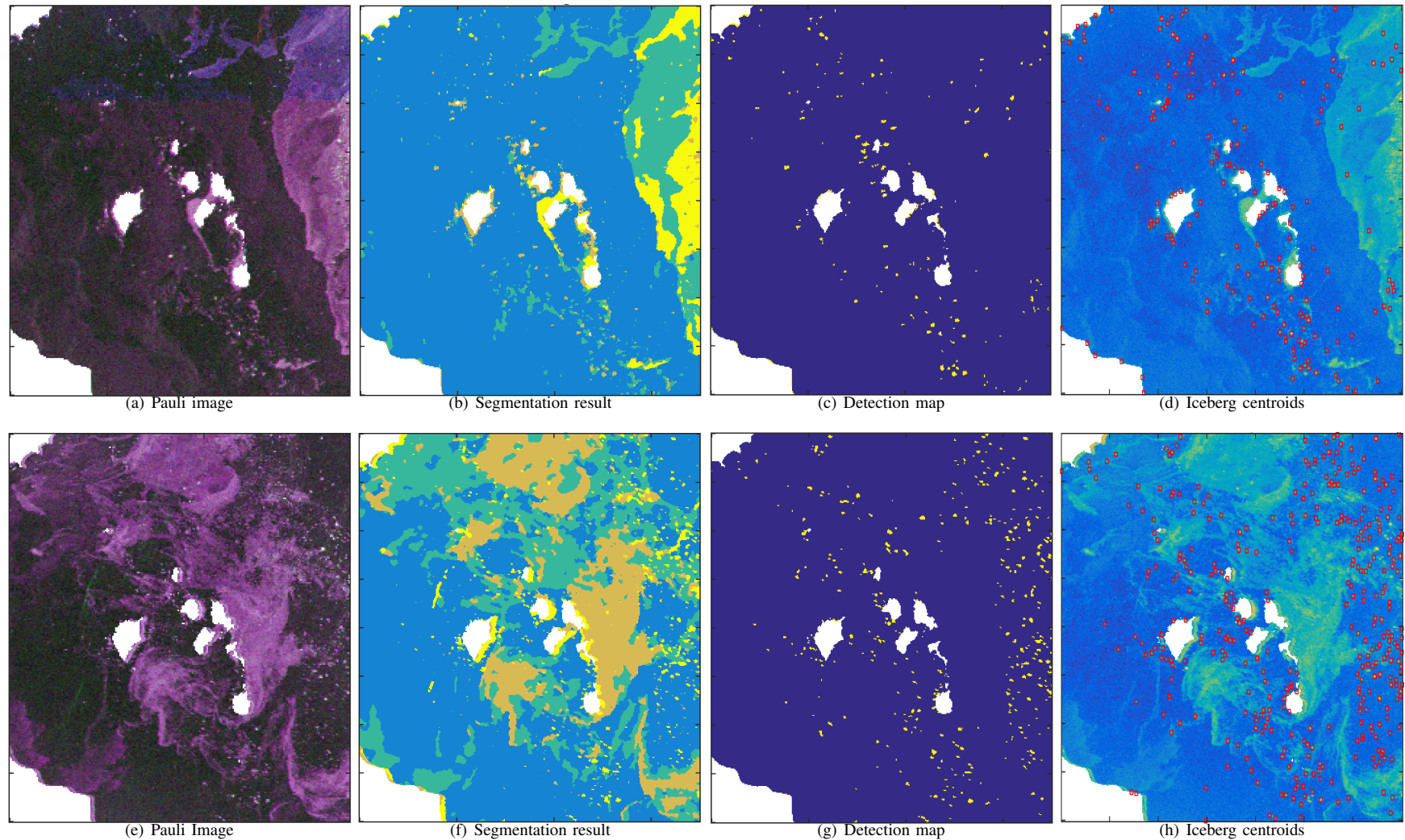

Fig. 3. Subsection of RS2 scenes acquired on April 19, 2015 (top row), and April 20, 2015 (bottom row), respectively, around the archipelago Lovenøyane in the inner fjord. (a) and (e) Pauli Images of subsections. (b) and (f) Segmentation results produced by the segmentation processor. (c) and (g) Detection maps. (d) and (h) Centroids of icebergs extracted from the segments in (c) and (d) on the geometric brightness images. 